\title{
THE PRECISE MEASUREMENT OF X-RAY DOSAGE ${ }^{1}$
}

\author{
By Lauriston S. Taylor
}

\section{ABSTRACT}

A comparison of the unit of X-ray dosage was made by Behnken and showed a variation of 4 per cent between various laboratories. For the purpose of definition and constancy a well controlled direct-current voltage source is provided for the X-ray tube. Using this, a comparison was made between the various methods using the same equipment for all. A large parallel plate ionization chamber was used to measure the $r$ unit as defined by international agreement. The discrepancies were found to be due to the electrostatic field distortion introduced by the various ionization current measuring devices. For the purpose of measuring $r$ precisely a null method is used and described. Sources of error are discussed and their probable magnitude indicated in certain cases. Under known conditions the difference between Behnken's and Glasser's determination has been duplicated in order of magnitude and in direction. A complete description of the Bureau of Standards equipment for the standard measurement of Röntgen dosage is given.

\section{CONTENTS}

I. Introduction

II. X-ray generating equipment. 772

III. Ionization measuring system

IV. Results . . .

V. Accuracy of measurements _.

\section{INTRODUCTION}

With the increase in the use of $\mathrm{X}$ radiation in medical therapy it has become more important to be able to express with some exactitude the actual amount of radiation being received by a patient. It is also necessary that this quantity be expressed in a common unit which is readily reproduced and in which there is no ambiguity.

In practice there are three distinct operations necessary in order to describe the radiation at a given location. (1) The measurement of the radiation intensity by means of a dosage meter. (2) the calibration of the dosage meter against a standard which measures the unit of $\mathrm{X}$ radiation exactly. (3) A determination of the wave-length

1 Read before the Radiological Society of North America, Dec. 5, 1928. 
distribution of the total radiation. It is with the second part that the Bureau of Standards is particularly interested and more especially with the exact measurement of the unit which was defined at the Second International Congress of Radiology at Stockholm, July, 1928, as follows: * * * "the quantity of radiation which, when the secondary electrons are fully utilized and the wall effect of the chamber is avoided, produces in one cubic centimeter of atmospheric air at $0^{\circ} \mathrm{C}$. and $76 \mathrm{~cm}$ mercury pressure such a degree of conductivity that one electrostatic unit of charge is measured at saturation current." This unit is called the "Röntgen" and is designated by small " $r . "$

In a recent paper Behnken ${ }^{2}$ gives the results of his comparison of the unit between the laboratories of Grebe, Küstner, Holthusen, Duane, Glasser, and the Physikalisch Technischen-Reichsanstalt. According to his measurements there is a discrepancy of about 4 per cent between various laboratories. No explanation for these differences has been given, and it is assumed that they are traceable to experimental error. However, as a result of some preliminary measurements made at the Bureau of Standards with the cooperation of Doctor Behnken, it was evident that improper electrostatic field conditions within the ionization chamber could account for at least some of the variation. To test this, a single ionization chamber was used under the same X-ray conditions but with various combinations of potentials applied to the plates as shown below.

\section{X-RAY GENERATING EQUIPMENT}

During the above-mentioned comparisons of Behnken it was demonstrated very clearly at the Bureau of Standards that, from the point of view of regularity of action, a mechanically rectified system was unsuited to precise X-ray measurements.

The wave-length energy distribution in the X-ray continuous spectrum excited by constant voltage can be expressed mathematically ${ }^{3}$ and can be determined experimentally with fair precision ${ }^{4}$ as a function of the X-ray tube voltage and other factors, such as target material, etc. The total energy varies approximately as the square of the voltage. Consequently, with a tube potential fluctuating in an uncertain manner-as the mechanical rectified caso-the energy distribution with time becomes uncertain; and, due to the shifting of the short wave length cut-off voltage, the quality is uncertain, since the spectral range is constantly shifting. As a result it was decided to use a kenetron rectified direct current source of high potential in which the voltage fluctuation or ripple was a minimum.

${ }^{2}$ H. Behnken, Strahlentherapie, 29, p. 192; 1928.

${ }^{3}$ H. Behnken, Zeit. fur Phys., 4, p. 241; 1921.

${ }^{4}$ T. Ulrey, Phys. Rev., 11, p. 401; 1918 
Thus there is a constant energy distribution and constant quantum cut-off within narrow limits.

This must not be confused with the medical application, for Coolidge has shown ${ }^{5}$ that for the requirements of medical treatment the direct and alternating high voltage source are about equally effective.

The circuit used is of the Hull ${ }^{6}$ and Webster and Henning ${ }^{7}$ type shown in Figure 1. The main transformer $T_{1}$ has one end of the secondary $S$ grounded. The other end goes to the cathode and anode, respectively, of two 250,000-volt kenetrons of the newest type. The filament current for these is supplied by separate insulation transformers $T_{2}$. The condensers $C_{1}$ and $C_{2}$ are of the Meirowsky Pertinax type in single units of $0.05 \mu \mathrm{f}$ each, thus avoiding the difficulty of series condensers. The two chokes $L_{1} L_{2}$ have 12,000 henrys induc-

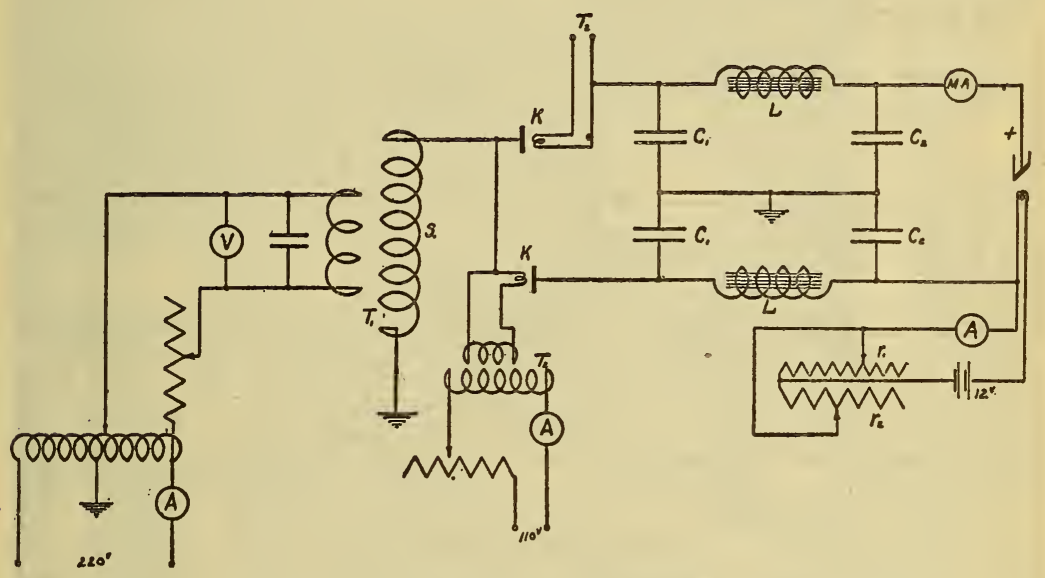

Fig. 1.-Diagram of $X$-ray generating system showing controls

tance and are wound on closed iron cores immersed in oil. Protective sphere spark gaps are placed across the kenetrons $K$, condensers and $\mathrm{X}$-ray tube.

The voltage is measured ordinarily by means of a large sphere gap, but an electrostatic voltmeter is available for control. An X-ray tube of the Coolidge $200 \mathrm{k}$. $\nabla$. deep therapy type has its filament current supplied from storage batteries placed in a coronaless copper case and in which the current is adjusted by remote control. The high-tension system is of three-fourths-inch tubing carefully installed to eliminate any corona discharge with consequent unsteadiness. It was found unnecessary to have any corona to act as a "safety valve," since the circuit design eliminated the likelihood of severe surges. A

${ }^{5}$ Am. J. Röent., 9, p. 77; 1922.

${ }^{6}$ A. W. Hull, Am. J. Röent., 2, p. 153; 1915.

7 P. L. Webster and A. E. Henning, Phys. Rev., 21, p. 301; 1923. 
60 -cycle $25 \mathrm{~K}$. V. A. synchronous motor generator supplies all of the transformers in order to obtain best control. A carefully adjusted overload circuit breaker in the main transformer primary prevents the possibility of damage from any heavy current discharges in the system. It has not been found necessary to use any protective resistances next to the X-ray tube, as no evidence of gas has been shown with the tubes used.

When making precise measurements a second operator controls the filament current and primary voltage. Under good conditions a precision of observation of 0.2 to 0.3 per cent can readily be obtained

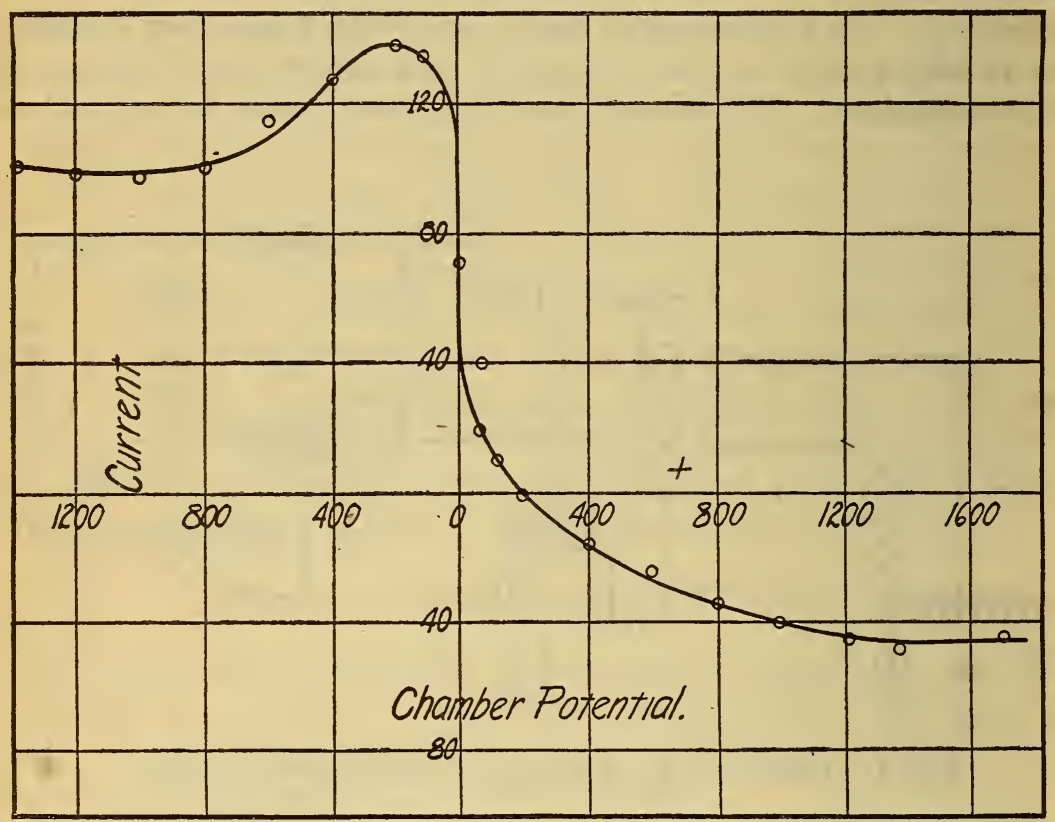

FIg. 3.-Curve showing ionization current as a function of the chamber potential, using early model Duane chamber and Victoreen electroscope

over a period of several hours. The variation from day to day, however, is frequently found to be double this amount.

\section{IONIZATION MEASURING SYSTEM}

It was decided from the start to use a large parallel plate ionization chamber as suggested by Duane ${ }^{8}$ in his early work. The first form of Duane's chamber was not of sufficient size, and the guards were not properly placed. Using such a chamber at the bureau with the cooperation of Doctor Behnken, mentioned above, the ionization current curve shown in Figure 3 was obtained where the current is

${ }^{8}$ Wm. Duane, Am. J. Röent., 9, p. 467; 1922. 
B. S. Journal of Research, RP56

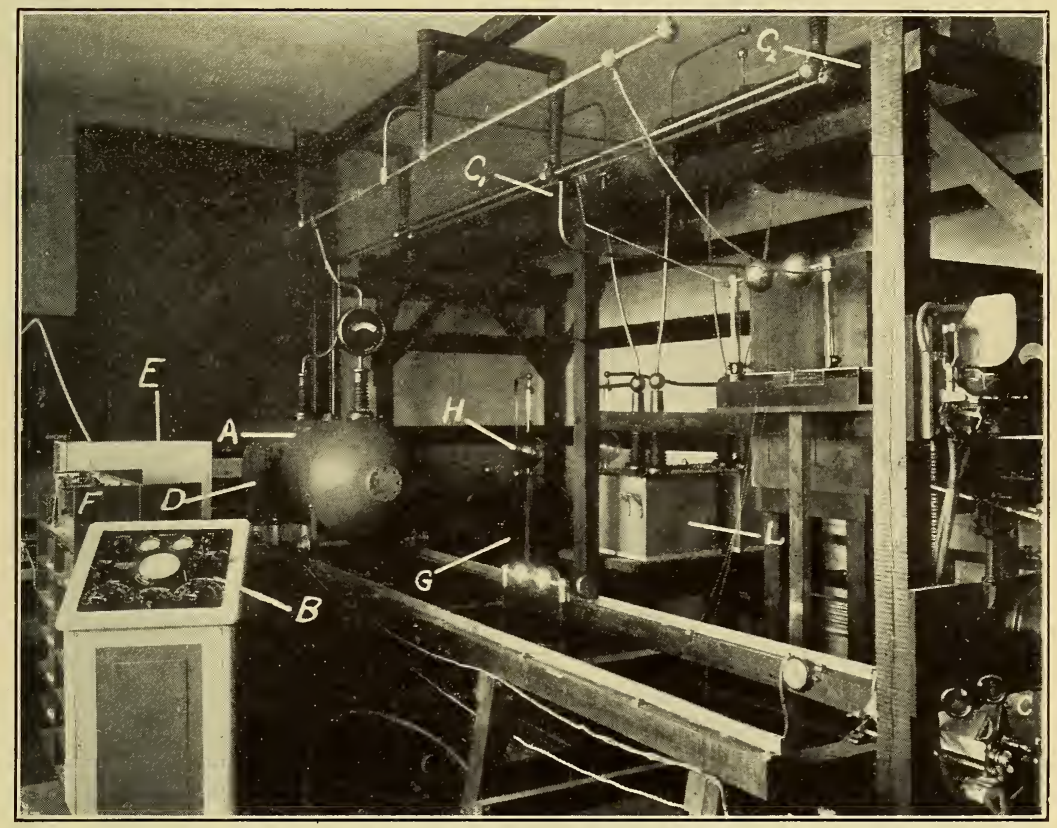

Figure 2.--General assembly of constant potential generator

$L$, Choke coils; $C_{1}, C_{2}$, condensers; $A$, one-quarter inch lead tube shield; $B$, control panel; $D$, magnetic shutter; $E$, ionization chamber; $F, 1,500$-volt battery; $G$, main transformer; $H$, kenetrons. 
B. S. Journal of Research, RP56
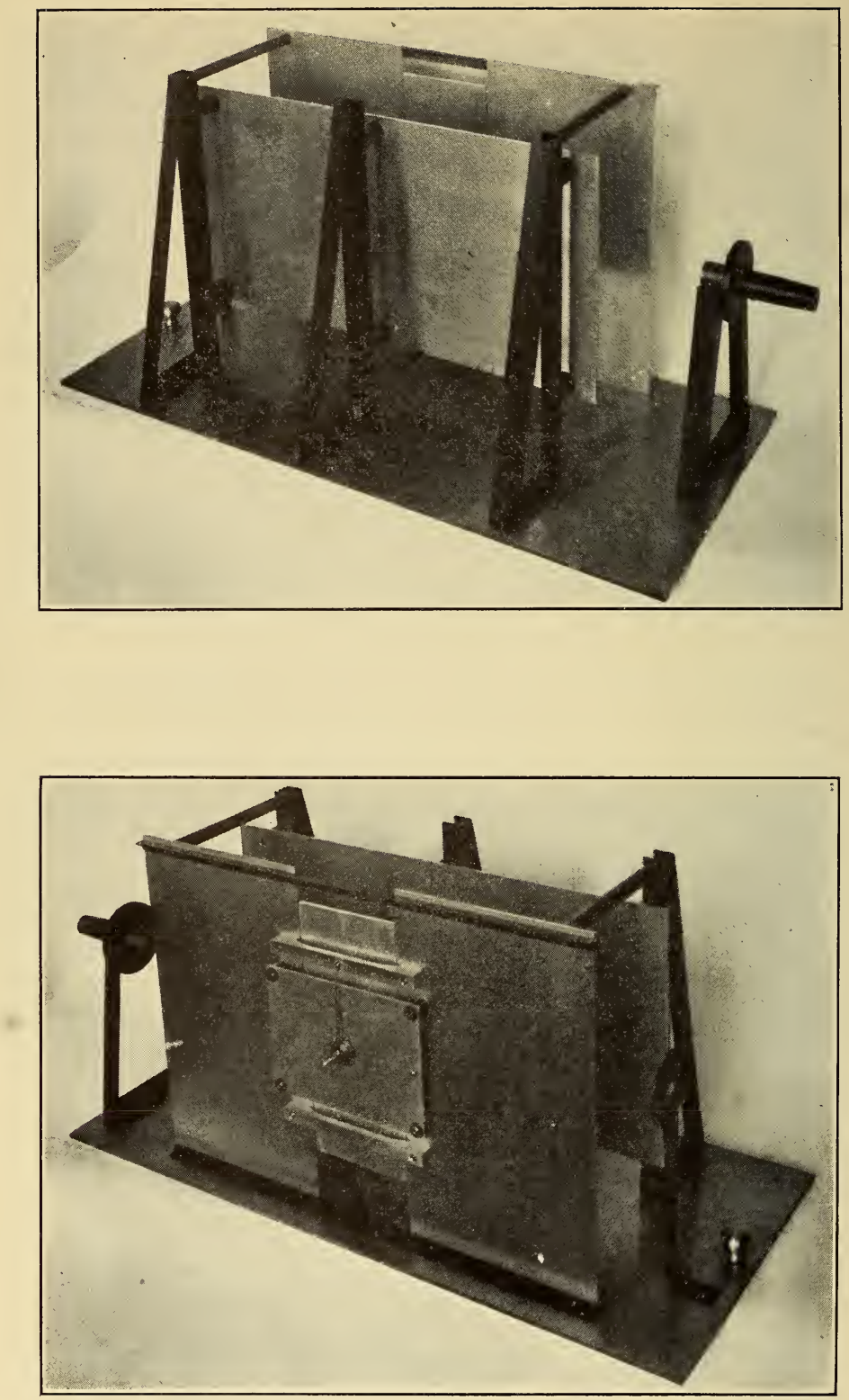

Figure 5.-Photographs of condenser plates composing the standard ionization chamber 
plotted as a function of the chamber voltage. By analysis of this curve some approximate idea can be obtained of the field conditions within the chamber. This curve is shown because it is quite typical for a faulty system where the electrode potentials differ and was present in an early type of chamber when used with an idiostatic electrometer in place of a galvanometer.

To avoid this difficulty, a null electrostatic measuring method is used. This has the advantage of being quite sensitive and being applicable to studying the field distortion as shown below. The galvanometer gives equally reliable results but does not have a sufficient range of sensitivity in the particular system.

The final form of standard chamber is shown in Figures 4 and 5. The plate spacing is $10 \mathrm{~cm}$. The measuring electrode is $10 \mathrm{~cm}$

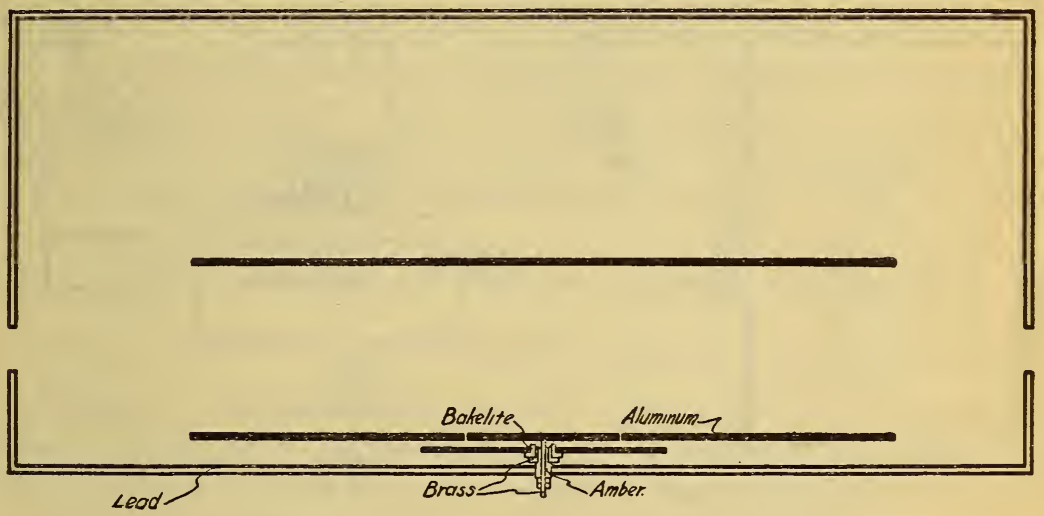

Frg. 4.-Cross section of ionization chamber

wide and the guards $20 \mathrm{~cm}$ wide with a $0.5 \mathrm{~mm}$ spacing between. The collector electrode is supported from the back by four amber posts bearing an aluminum yoke spread between the guards. All of the electrodes are mounted on a brass plate as shown in the photograph (fig. 5). The guard plates are ordinarily earthed. The entire electrode system is placed in a one-eighth inch lead box with a one-fourth inch lead front. The spacing between the high-voltage electrode and the case was never less than the distance across the condenser normal to the beam $(10 \mathrm{~cm})$. In the present chamber it is about $15 \mathrm{~cm}$. Since the assembled unit is very large and heavy, it is mounted on an adjustable track so as to be readily aligned. Behind the ionization chamber is a lead chamber for preventing as much radiation as possible from escaping into the room. There is no measurable back scattering from this chamber. 
The diaphragm system is arbitrary, being approximately the same. as used by Behnken ${ }^{9}$ and Glasser. ${ }^{10}$ More recent work by Failla ${ }^{11}$ has indicated that these diaphragm systems are perhaps not exactly correct. In any case it was not altered throughout the entire experiments. About $1 / 2 \mathrm{~cm}$ from the X-ray tube wall is placed a disk of 55 per cent lead glass $2 \mathrm{~cm}$ thick and having in the center a $12 \mathrm{~mm}$ hole. The position of this diaphragm is adjustable in all directions.

The tube is contained in a one-fourth inch lead cylinder lined with steel for rigidity. This is mounted on a 16 -foot track so as to move into several positions.

An electrically operated lead shutter is mounted on the tube shield and controlled from the observer's position. The shutter system is similar to that used by Webster ${ }^{12}$ and others, and the timing is
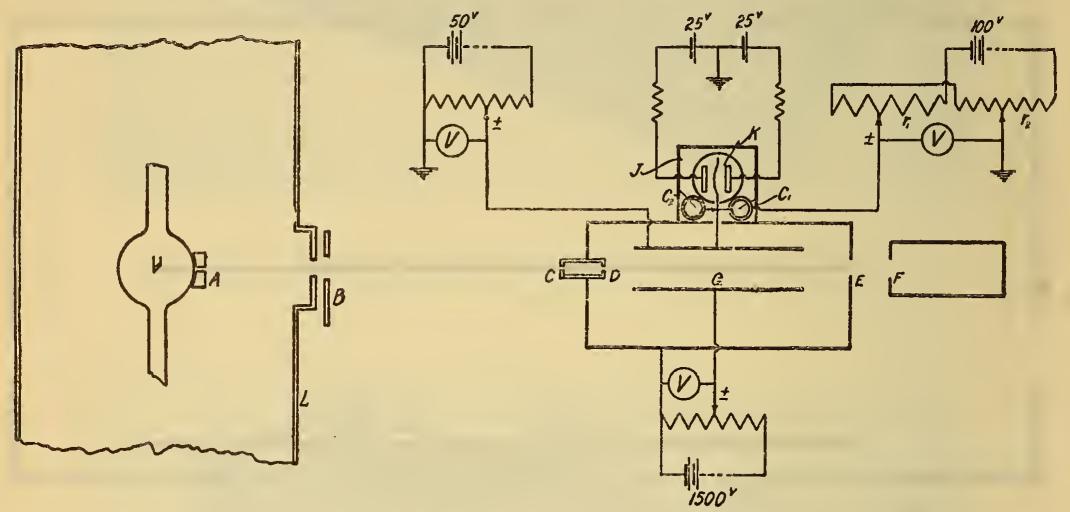

FIG. 6.-Diagram of ionization chamber system

$A$, lead glass diaphragm. $B$, diaphragm and shutter on tube shield. $C$, diaphragm for defining the standard beam. $D$, diaphragm to reduce scattering of radiation within the chamber. $E$, diaphragm at back of lead box. $F$, lead box to stop radiation from entering the room. $G$, ionization chamber. $J$, one-eighth inch lead box surrounding electroscope system. $K$, LutzEdelmann Wollaston fiber electrometer. $L$, cylindrical X-ray tube shield

independent of any uncertain electrical contacts. The shutter can be opened for any multiple of 7.5 seconds to within at least $1 / 1,000$ second and avoids the use of a stop watch.

Figure 6 shows the electrostatic measuring system. The electrometer and condensers are in a one-eighth inch lead box fastened to the chamber box. This provides electrostatic and X-ray protection. The system was carefully tested and all measurable leakage eliminated. $K$ is a Lutz-Edelmann fiber electrometer, which for the null readings was connected as shown with \pm 25 volts on each knife-edge. $C_{1}$ and $C_{2}$ are amber insulated concentric cylinder condensers, and

${ }^{9}$ H. Behnken, Strahlentherapie, 26, p. 79; 1927.

10 O. Glasser, Am. J. Röent., 19, p. 47; 1928.

is $\mathrm{G}$. Failla (seen in manuscript form).

12 D. I. Wobster and A, E. Henning, Phys, Rov., 21, p. 301; 1923. 
$C_{2}(140 \mu \mu \mathrm{f})$ can be cut in and out of the system from outside the shield. For the purpose of null reading, on one side of $C_{1}$ a variable potential is applied by means of the coarse and fine rheostats $r_{1}$ and $r_{2}$. This is read on a precision voltmeter.

In making observations the electrode is first ungrounded and the shutter then opened. An observer maintains the cross hairs on the zero potential mark by continuously adjusting the rheostats $r_{1}$ and $r_{2}$ during the entire exposure. Thus, the potential of the collector electrode never differs from that of the guards by more than a small amount, which is determined by the sensitivity of the electrometer and the capacity of the system. In the particular case it was \pm 0.3 volt.

In such a method the potential necessary to counteract a given potential increase of the system depends solely upon the geometrical configuration of the system and is independent of the electrometer sensitivity. The electrometer sensitivity only limits the divergence of the collector potential from zero as shown above. A theoretical constant $k$ of the apparatus depending upon this configuration has been found experimentally to hold constant as long as the capacity of the system is unchanged.

If a change of potential $\Delta V$ between plates of the condenser $C_{1}$ produces a change $\Delta v$ on the insulated system, then $\frac{\Delta V}{\Delta v}=k_{\mathrm{c}}$ when the condenser $C_{1}$ only is in the system. If in a time $t$ it is necessary to apply a potential $V$ to the condenser $C_{1}$ to maintain zero potential on the insulated system, then the corresponding charge $Q$ in e. s. $u$. due to the ionization is then given by

$$
\begin{aligned}
Q & =\frac{C V}{k_{\mathrm{c}}}(V \text { in e. s. u. }) \\
& =0.9 \frac{C V}{300 k_{\mathrm{c}}}(V \text { in volts and } C \text { in } \mu \mu \mathrm{f})
\end{aligned}
$$

where $C$ is the total capacity of the system. Thus, under standard conditions of temperature and pressure, the dose in $r$ per minute is given by

$$
r / \min =\frac{0.9 \times C V \times 60}{300 \times t k_{\mathrm{c}} W}
$$

where $W$ is the effective volume of ionized air and $C$ is measured in micromicro farads. Including next, the approximate correction for temperature $T$ (degrees absolute) and pressure $P(\mathrm{~mm})$

$$
r / \min =\frac{0.9 C V \times 60 \times 273 \times \mathrm{P}}{300 \times i k_{\mathrm{c}} \bar{T} \mathrm{~T} \times 760}
$$


The constant $k_{\mathrm{c}}$ was determined carefully by charging the electroscope first directly from a potentiostat and then to the same scale points by means of the compensating potential applied to the condenser $C_{1}$. The curves in Figure 7 give the experimental relation between $V$, the compensating potential, and $v$, where $k$ is given by

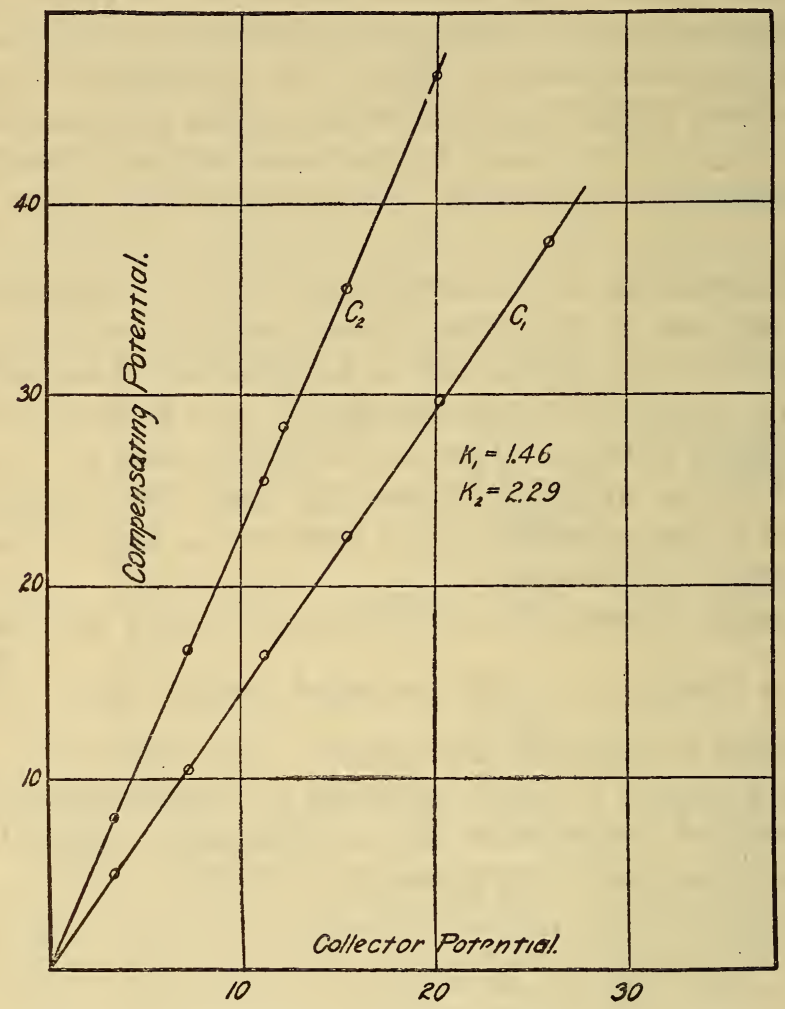

FIG. 7.-Curves used for the determination of the apparatus constant " $k$ "

the slope. In the particular case only two capacities were used, but it is noticed that the relation is closely linear, and by taking the best line through the points a very accurate determination of $k$ is possible. In addition, this was tested, using a standardized variable condenser outside the case and the same accuracy was found. In the expression (4) above the ratio $\frac{C}{k_{\mathrm{c}}}$ should be a constant for all capacities. This was found to be true over a wide range of electrometer sensitivities and from day to day. Thus the error introduced in $r$ by certain other measuring methods due to the measurement of the volt sensitjvity of the electrometer is eliminated. 


\section{RESULTS}

The discrepancies between the various measuring methods is at once apparent from the saturation curves obtained by the same chamber under constant X-ray conditions. Four such curves are shown in Figure 8. In all cases the chamber potential was positive. (a) The electroscope was charged negatively and the discharge time from -50 to -32 volts measured. The saturation value was 3.55 $r / \mathrm{min}$. (b) The electroscope was charged positively and the charge time from +32 to +50 volts measured. The saturation value was $2.77 \mathrm{r} / \mathrm{min}$. (c), the electroscope was again charged positively and charging time from +50 to -50 measured. (This can be considered

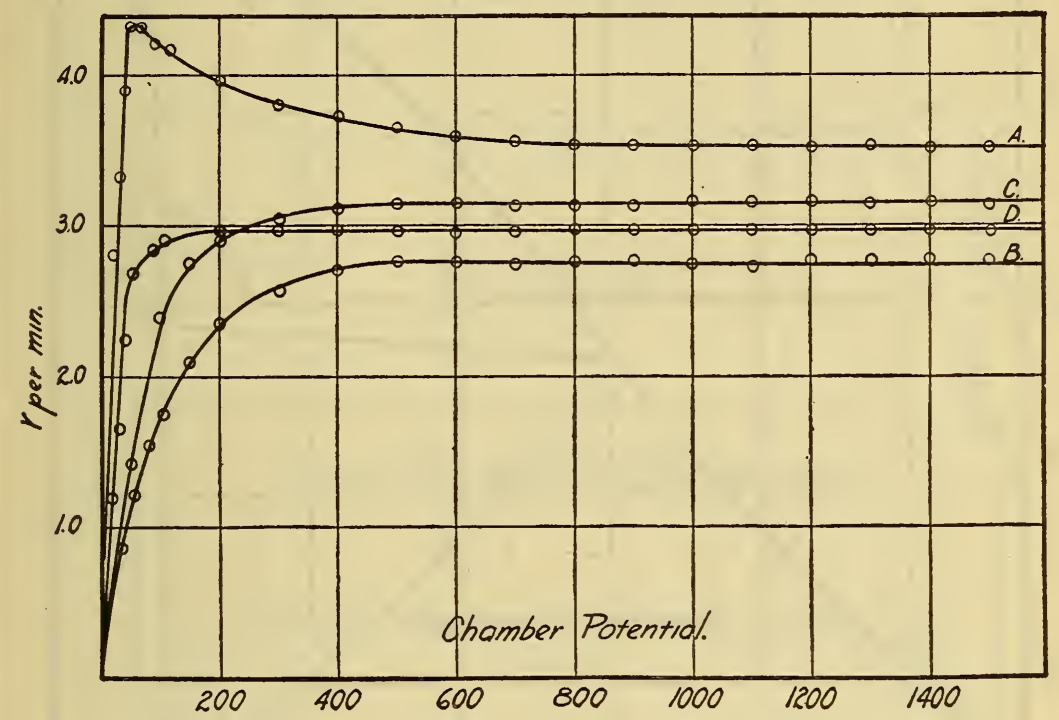

FIG. 8.-Saturation curves for standard ionization chamber using different measuring methods

$A$, using a discharging, fiber electrometer. $B$, using a charging, flber electrometer. $C$, using an idiostatic fiber electrometer. $D$, using a null reading fiber electrometer

as an average of $a$ and $b$.) The saturation value was $3.15 \mathrm{r} / \mathrm{min}$. (d) The null reading method gave a saturation value of $2.93 \mathrm{r} / \mathrm{min}$. (e) Galvanometer method. (For the low energies used it was not practicable to determine the whole curve with the galvanometer, but above saturation it agreed within the experimental error with the null method.)

Field distortion (to be described later) in the first two methods can account for the peculiar form of the curves. Glasser apparently recognizes this and then assumes that the distortion shown by curves $A$ and $B$ is equal and opposite, since he essentially uses method C. Since, however, method C produced a higher saturation value than 
$\mathrm{D}$ and $\mathrm{E}$, the actual field distortion was carefully determined in the present case by experiment.

To study this two methods were used which agreed within experimental error. With the chamber potential positive and well above saturation, the guard plates were set at zero potential and the electrometer system charged to various potentials from -45 to +45 volts. At each voltage value $r$ was measured by the null method, maintaining the potential difference between guards and collector constant to within \pm 0.3 volts. Since $r=$ const $\times V$ (equation (4)), a curve

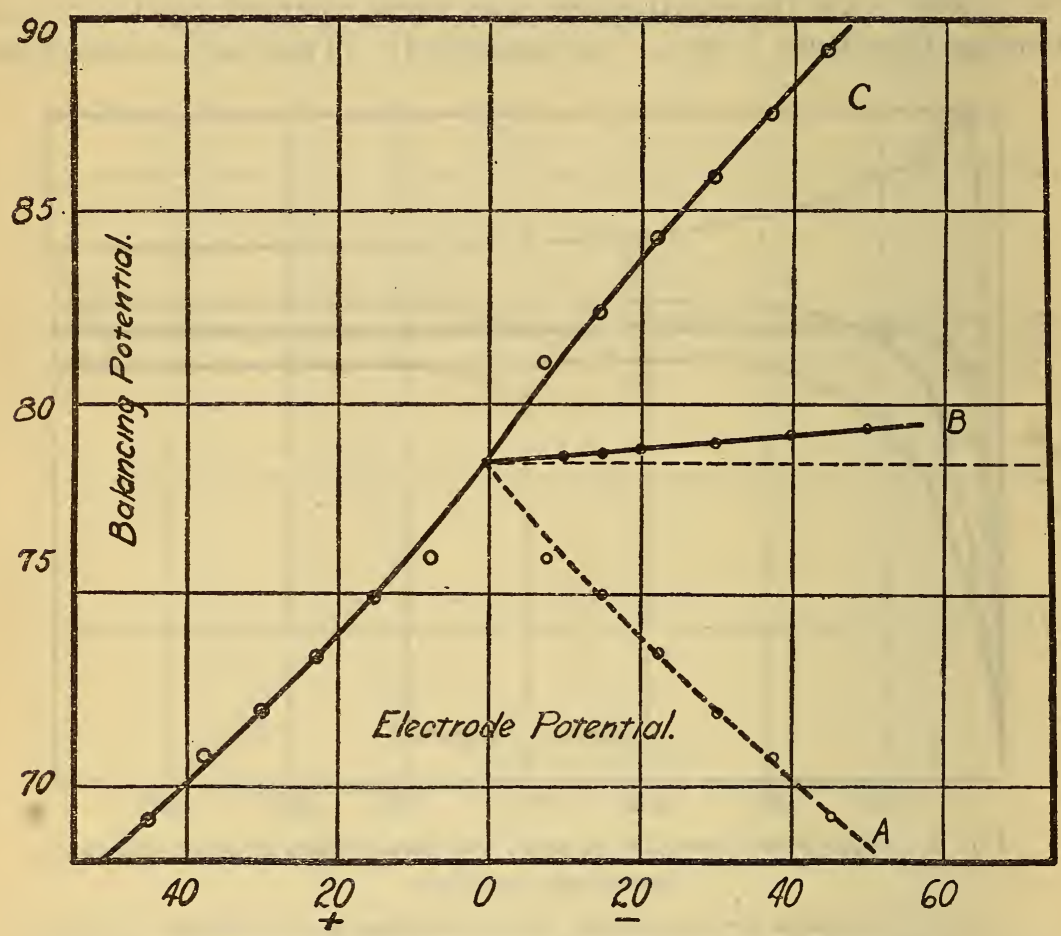

Fig. 9.-Saturation current in the standard ionization chamber as a function of the potential difference between the guard plates and collector plate

Curve $B$ shows the average saturation current for corresponding positive and negative potential differences. (Balancing potential is proportional to the saturation current)

showing $V$ plotted against the potential difference between plates will give the difference in $r$ according as the potential of the collector is above or below the guard. This is given by $C$ in Figure 9 . The value of $r$ by the null method is that where the curve crosses the axis (78.4 volts in the particular case). The broken line $A$ is simply the positive portion of the curve swung under the negative portion for more obvious comparison. Averaging these values it is seen at once from curve $B$ that the mean falls consistently above the value determined by the null method. In other words, the distortion pro- 
duced when the collector is negative is greater than that when the collector is positive with respect to the guard potential. A picture of the field is given in Figure 10 for each case.

Exactly the same result was obtained when the collector was maintained at zero potential and the guards are set at the same potentials as above.

It may be said that the irradiated volume is determined by the cross section of the beam and the bounding lines of force that just reach the collector at the edges. Thus it has been shown that due
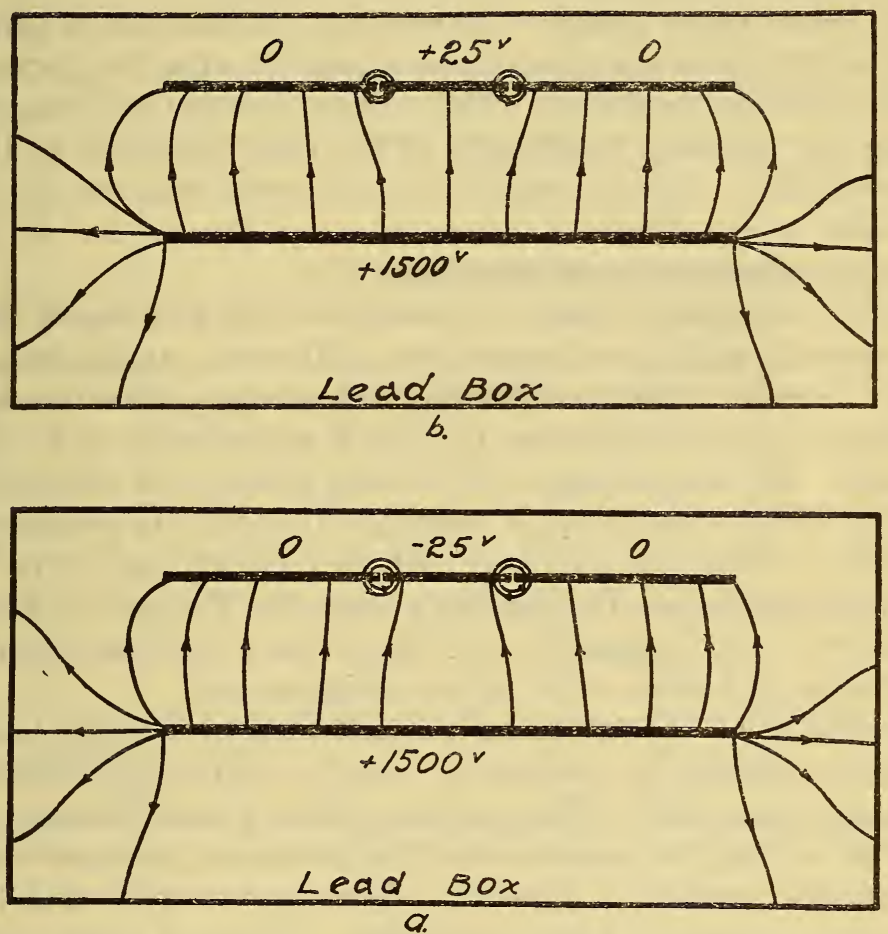

FIg. 10.-Probable electrostatic field diagram in the standard chamber

$A$, when using a discharging electrometer. $B$, when using a charging electrometer

to controlled field distortion the length of irradiated volume varies in an unsymmetrical manner, the degree of dissymmetry depending upon the geometrical configuration of the system. This effect should be symmetrical when the width of the collector and the width of the guards are both very great with respect to the spacing of the condenser plates.

Referring again to Figure 9 we may regard the curves $A$ and $C$ as defining the irradiated volume limits for different potential differences between guard and collector. The average curve $B$ then indicates 
the change in length of the irradiated volume and the corresponding error introduced by a potential difference between the guard and collector, such as caused by the use of an idiostatic electrometer.

Rosa and Dorsey, ${ }^{13}$ in their work with precision guard plate condensers, have calculated the error due to a very small displacement between the guards and collectors if their potential differs. A measurable error of this kind was found by displacing the collector plate in the present ionization chamber when using the deflection method of measurement. It is not likely, however, that this error has been of any large amount in the work of others.

In a recent paper Duane ${ }^{14}$ reported that the saturation current for a similar ionization chamber decreased slightly after the plate spacing reaches a certain maximum. This could be avoided in his chamber by placing the shielding diaphragm at the same potential as the high potential plate. This is avoided in our present chamber by spacing the shield at a sufficient distance from the electrodes. G. Failla ${ }^{15}$ has independently substantiated this.

In the comparison made by Behnken ${ }^{16}$ it was found that the Reichsanstalt unit agreed with that of Duane. An analysis of the method used by Behnken shows that there is only a fraction of a volt difference of potential between the guards and collector in his pressure chamber. His disagreement with Glasser's value is in the same direction and of the same order of magnitude as found in the present experiments. Thus, it is indicated that the methods used by the Physikalische-Technischen Reichsanstalt and the Bureau of Standards should be in good agreement and should each yield an accurate determination of $r$ when other errors are eliminated.

An analysis of the measuring method used by Glasser, ${ }^{17}$ and as a secondary method by Behnken, ${ }^{18}$ can be made experimentally by two simple operations. The guards are set at a fixed potential of such value as to bring the electrometer fiber, when at the same potential, to a sensitive position on the scale. The electroscope is then charged to a value somewhat higher than this. Times of transit are then measured with a split second stop watch between every five divisions on the scale. The voltage sensitivity curve of the electrometer is shown in $A$ (fig. 11) and is seen to be continuously changing. The point $a$ on the sensitivity curve corresponds to the guard plate potential (147 volts). Points $b$ and $c$ correspond to equal voltage steps above and below $a$, through which range the electrometer was discharged; $b^{\prime}$ and $c^{\prime}$ are the corresponding time points. The corre-

\footnotetext{
13 E. B. Rosa and N. E. Dorsey, B. S. Bull., 3, p. 433.

14 Wm. Duane, Am. J. Röent., 19, p. 461; 1928.

1s See footnote 11, p. 776 .

16 See footnote 1, p. 771.

17 O. Glasser, Am. J. Röent., 19, p. 47; 1928.

${ }^{18}$ H. Behnken, Strahlentherapie, 26, p. 79; 1927.
} 
sponding transit time curve $B$ for the electrometer discharging is given below and is also seen to be continuously varying. The formula used in calculating (4) from this data is given by

$$
r / \min =\frac{C \times V \times 60}{300 \times W \times t}
$$

where $V$ in this case is the volt sensitivity. From the curves above the ratio $\frac{V}{t}$ can be obtained in any suitably small increments. Thus, in order to determine $r / m i n$ accurately the ratio $\frac{\Delta V}{\Delta t}$ should be either constant or linear with respect to the scale position. However, in the

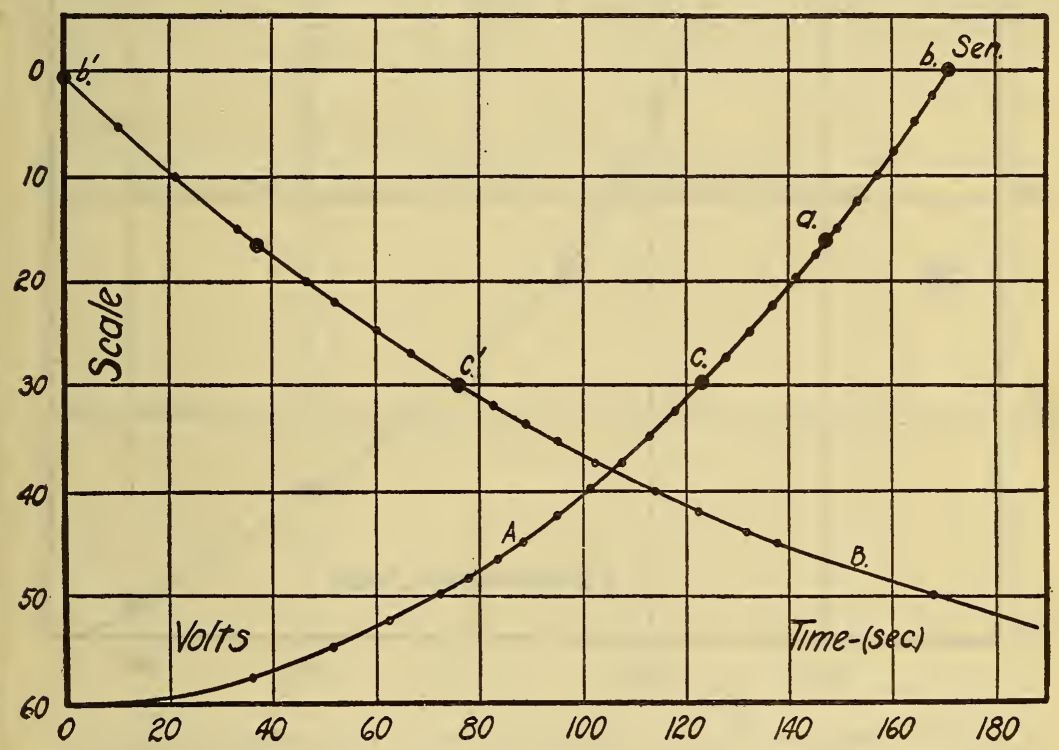

FIG. 11.-Sensitivity curve and time-transit curve for idiostatic measuring method

particular case the ratios obtained from the curves in Figure 11 when plotted against scale position gave a nonlinear relation as indicated in Figure 12. It is thus clear that under ordinary circumstances the above method will not yield an accurate determination of $r / \mathrm{min}$. It must be pointed out, however, that if a sufficiently small voltage range is used the error will not be large, since the relation is nearly linear for short ranges of voltage.

Also, it is probable that by varying the gap between collector and guards this nonlinearity may be increased or diminished, thus changing the error introduced. When the above method of measurement is used, the error should be carefully studied and proper precautions be taken to eliminate it or reduce it to a minimum. 
There is yet another source of error detectable when using a comparatively highly charged collector or guard plates. A small amount of secondary radiation scattered by the air may actually pass between the guards and collector producing ionization both between the guards and collector and in the space behind them. In the first case the ionization current changes direction as the collector potential passes that of the guards. The error introduced is thus additive in one case and subtractive in the other. In the second case the ionization will be constant provided saturation exists behind the plates.

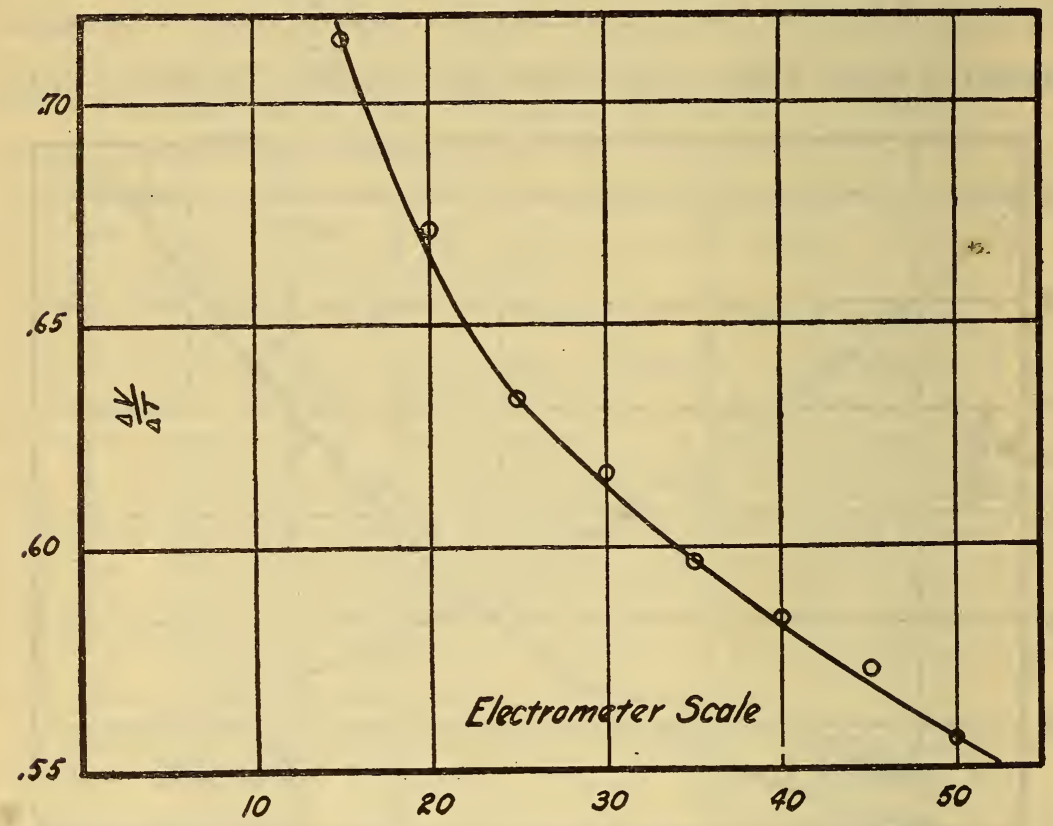

FIG. 12.-Relation of $\frac{\Delta V}{\Delta t}$ to $V$ (electrometer scale) using idiostatic measur-

When employing a null method in which the collector, guards, and box are all at zero potential, these errors will be absent.

\section{ACCURACY OF MEASUREMENTS}

The actual error in the present measuring system can be approximately determined. It must be emphasized, however, that an arbitrary diaphragm system has been used thus far and the error in determining the volume $W$ of air ionized has been omitted. For an accurate measurement of $r / \mathrm{min}$ this should, of course, be known.

The primary voltage on the high tension and filament transformers is measured to within \pm 0.1 volt. The ripple or fluctuation in the 
rectified voltage was calculated with sufficient accuracy ${ }^{19}$ and found to be approximately 0.2 per cent when a current of $1.0 \mathrm{~m}$. a. is being drawn. The X-ray tube current is controlled to within $\pm 0.005 \mathrm{~m}$. a.

When using the compensating potential the mean deviation of a number of readings is usually found to be 0.2 to 0.3 per cent. The voltage can be read to \pm 0.1 volt out of 100 volts, and the meter is accurate to 0.25 per cent. The capacity of the system is measured by means of a bridge calibrated at the Bureau of Standards. The capacitance can be determined with an accuracy of 0.5 per cent or better. By referring to the curves in Figure 6 for the determination of $k$, it was shown that the ratio $\frac{C}{k}=$ const. This also serves as a check on the capacitance measurements. It is thus seen that aside from the diaphragm error the accuracy in the determination of $r / \mathrm{min}$. is 1.0 per cent or better.

Washington, September 30, 1928.

${ }^{19}$ L. B. Jolley, Alternating Current Rectification, J. Wiley \& Sons; 1924. 